For Internal Distribution Only

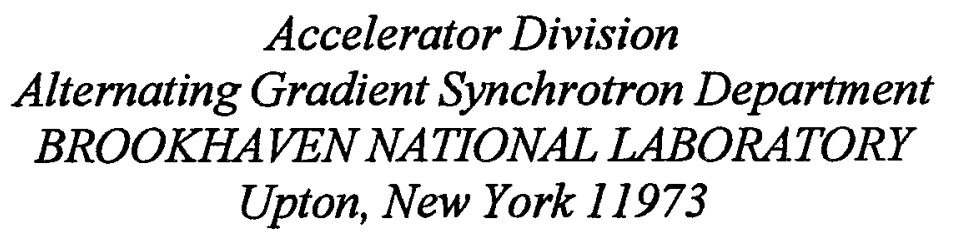

Accelerator Division

Technical Note

AGS/AD/Tech. Note No. 451

SPACE CHARGE EFFECT AND THE AGS INJECTION LOSS

S. Y. Zhang

October 29, 1996 


\title{
Space Charge Effect and the AGS Injection Loss
}

\author{
S.Y. Zhang
}

\section{Introduction}

It has been suggested [1] that the largest portion of the BTA transfer loss in high intensity proton mu is located in the AGS injection section. In this note, we show that the space charge induced tune spread might be a dominant factor in this loss.

The envelope equation [2], describing the space charge effect, predicts that when the maximum tune shift is depressed beyond the half integer resonance, or integer resonance, by a quarter of the shift, the envelope resonance will happen.

The simulations $[3,4]$ further show that under similar conditions, the beam emittance will blow-up.

Several experiments conducted on low energy proton synchrotrons [5-9] have confirmed the theory and the simulation.

Based on these results, the AGS high intensity proton injection is analyzed. The space charge tune spread, immediately after the bunched beam enters the AGS ring, is large enough for the envelope resonance and the emittance blow-up. Therefore, it is likely that the space charge effect is a dominant factor to cause significant beam loss in the AGS injection.

\section{Theory}

The defocusing space charge force can be shown as $k$ in the usual equation

$$
x^{\prime \prime}+K x=k x
$$

where $K>0$ is the external forcusing force.

The effect of space charge can be seen as resemble to the quadrupole error, or the halfinteger error. There are, however, two fundamental differences. Firstly, the space charge force as an internal force affects each other among the particles. This force is distributed among the particles unequally. Secondly, a strong space charge effect will produce larger beam size. In turn, a larger beam size necessarily implies to a weaker space charge effect, as shown in,

$$
k=\frac{\alpha}{\sigma^{2}}
$$

where $\alpha$ is a constant, and $\sigma$ is the beam size.

To deal with these problems, we let,

$$
x=\sigma \cos \phi
$$


where both the envelope $\sigma$ and the derivative of phase advance, $\phi^{\prime}$, are not constant. Substituting (3) into (1), we get,

$$
\sigma^{\prime \prime}-\frac{\epsilon_{0}^{2}}{\sigma^{3}}+K \sigma-k \sigma=0
$$

where $\epsilon_{0}$ is the beam emittance defined by the equilibrium beam size $\sigma_{0}$ as,

$$
\epsilon_{0}=\sqrt{K} \sigma_{0}^{2}
$$

Substituting (2) into (4), we get,

$$
\sigma^{\prime \prime}-\frac{\epsilon_{0}^{2}}{\sigma^{3}}+K \sigma-\frac{\alpha}{\sigma}=0
$$

which is the envelope equation.

Consider the Laslett tune shift calculated by $[10,11]$. Assuming symmetric beam, the tune shift is

$$
\Delta \nu=\frac{-N r_{0} R}{4 \pi \nu_{0} \beta^{2} \gamma^{3} \sigma^{2}}
$$

where $N$ is the total particle number, $r_{0}$ is the classical radius of proton, $R$ is the machine radius, and $\nu_{0}$ is the bare tune. Note that here $\sigma$ is the $r m s$ beam size. The coefficient $\alpha$ is associated with the tune shift $\Delta \nu$ by

$$
\alpha=\frac{-2 \nu_{0} \Delta \nu \sigma_{0}^{2}}{R^{2}}
$$

Substituting (8) into (6), an equation describing the envelope $\sigma$ with respect to the maximum tune shift $\Delta \nu$ is established. By analyzing this equation, Sacherer [2] found that the envelope resonance happens when the maximum tune shift $\Delta \nu$ is depressed beyond the half integer resonance by a quarter of the shift. For instance, let the AGS vertical tune at the injection be 8.85 , then the envelope resonance happens if the Laslett tune shift is larger than 0.47 .

We now give some comments on this result.

1. The accurate account of the space charge effect in terms of the tune spread is hardly dependable, given the complication of the problem. Qualitatively, however, the envelope equation analysis is very suggestive. The Laslett tune shift can be safely depressed beyond the half integer resonance by a certain amount of the shift! The envelope resonance happens only beyond that limit.

2. One reason of this is that the envelope resonance is relevant to the resonance modes, rather than to individual particles. Note that the Laslett tune shift is the maximum tune shift, which is relevant to only a small portion of the particles [11].

3. The envelope resonance can be understood as follows. When a certain amount of particles have tune shift larger than the half integer resonance, these particles perform betatron oscillation with large amplitude in terms of modes, due to the beta function distortion. Though this large amplitude oscillation is not the emittance blow-up in usual sense, it reduces the tune shift. In this way, envelope oscillation happens. 
4. In the envelope equation analysis, the quadrupole (half integer) error, as exciting force, is not essential. It does, however, affects the strength of the resonance, as seen from the envelope equation with the exciting force $f$,

$$
\sigma^{\prime \prime}-\frac{\epsilon_{0}^{2}}{\sigma^{3}}+K \sigma-\frac{\alpha}{\sigma}=f
$$

5. The emittance growth is not directly related to the envelope resonance. However, it is clear that the envelope resonance is a source of the emittance growth, given the machine imperfections.

6. Under weak envelope resonance, the beam loss can be understood by the beam scraping as the emittance approaches the machine acceptance. Under strong envelope oscillation, the turbulent resonance certainly implies large increase of the oscillation amplitude of some modes, which can cause massive beam loss within short period of time, accompanied by rapid emittance blow-up.

\section{Simulation}

In [3], simulation for LEB of SSC shows that the beam emittance grows when the bare tune becomes smaller than 11.66, for a fixed tune spread $\Delta \nu=-0.33$. Without half integer error, the emittance growth is weaker, however, the threshold of the emittance growth in terms of bare tune did not change.

In [4], simulation using the parameters developed from real machines shows similar results. Also can be seen from the simulation is that for the LEB, the major emittance growth is completed within $6 \mathrm{~ms}$. In the case of strong space charge tune shift, major emittance growth occurs in a period of about $2 \mathrm{~ms}$.

\section{Experiments}

The following is a summary of the experiments conducted on several low energy proton synchrotrons.

\begin{tabular}{||c|c||c|c|c|c||c||}
\hline \hline Parameter & Notation & PSR & CPS & PSB & FermiB & Unit \\
\hline \hline Kinetic Energy & $E_{k}$ & 0.797 & 1.0 & 0.05 & 0.204 & $\mathrm{GeV}$ \\
\hline Radius & $R$ & 14.34 & 99.78 & 25.0 & 75.5 & $m$ \\
\hline Number of Particles & $N$ & 23 & 23 & 6 & 2 & $10^{12}$ \\
\hline Bunching Factor & $B_{f}$ & 0.4 & 0.32 & 0.44 & 0.39 & \\
\hline Emittance & $\epsilon_{N, 95 \%}^{y}$ & 83.7 & 37.8 & 43.3 & 12.0 & $\pi \mu m r$ \\
\hline$r m s$ Beam Size & $\sigma_{y}$ & 7.75 & 7.44 & 10.0 & 5.80 & $m m$ \\
\hline Vertical Tune & $\nu_{y}$ & 2.14 & 6.28 & 5.45 & 6.80 & \\
\hline Tune Spread & $\Delta \nu_{y}$ & 0.18 & 0.37 & 0.66 & 0.38 & \\
\hline \hline
\end{tabular}

Table 1 
In Table 1 , the tune spread is calculated by

$$
\Delta \nu=\frac{-N r_{0} R}{4 \pi B_{f} \nu_{0} \beta^{2} \gamma^{3} \sigma^{2}}
$$

which is modified from (7) by adding the bunching factor $B_{f}$. Sometimes it is convenient to use the normalized emittance to calculate the tune spread, as shown in [11],

$$
\Delta \nu=\frac{-3 N r_{0}}{2 \pi B_{f} \beta \gamma^{2} \epsilon_{N, 95 \%}}
$$

Some other details are listed as follows.

PSR [5]: The tune shift shown in Table 1 is calculated using the $r m s$ beam size given in [5]. The calculated tune shift is depressed beyond the integer by $22 \%$ of the shift.

The experiments had been exercised for different intensities and different bare tunes. In all cases, the emittance grows along with either the intensity increase or moving the bare tune to approach the integer resonance. Thus, the resulted incoherent tune shift is depressed beyond the integer by a fixed amount.

In this experiment, some error exists in terms of beam size. The beam yertical profile is distorted due to the mis-steering injection, which causes 'smoke-ring' particle distribution in phase space.

CPS [6,7]: The SPS fixed target program is used, and the tune spread is depressed beyond the integer by $24 \%$ of the tune shift.

The experiments were set up by changing the bunch length to affect the tune spread. For shorter bunches, however, larger emittances are observed, which keep the amount of the tune spread beyond the integer line constant. Another set of experiments with the bare tune of 6.22 gives rise to same consistent results.

PSB [8]: Some data presented in [8] are questionable. Therefore, we simply use more realistic rms beam size as $\sigma_{y}=10 \mathrm{~mm}$, and use a more comfortable bunching factor $B_{f}=$ 0.44 . The resulted tune spread is depressed beyond the integer by $32 \%$.

The PSB was operated by filling the ring acceptance, and there was significant beam loss. Other factors affecting the tune spread are that the beam horizontal emittance is triple of the vertical one, and the debunchers in the Linac transfer line were used to manipulate the momentum spread, which improves the bunch shape.

FermiB [9]: The experiments were exercised for beam intensities from $N=10^{12}$ particles to $N=3 \times 10^{12}$ particles, and the emittance is enlarged accordingly. The tune shift is kept at a constant -0.38 . Since the vertical tune is at 6.8 , the tune spread is depressed beyond the half-integer by $21 \%$ for all intensities.

As conclusion, in all these machines, consistent results have been obseryed, which agree with the prediction of the theory of the envelope oscillation and the simulation. When the tune spread is increased, the beam emittance is not affected until the tune spread is depressed over the half integer or integer resonance by a certain amount. Beyond that limit, the emittance will blow-up. In these experiments, the maximum tune shifts have been found beyond the half integer or integer resonance by $21 \%$ to $32 \%$ of the tune shift. The Laslett tume shift is then self-stabilized by the associated emittance growth. 


\section{$5 \quad$ AGS Injection Loss}

In 1996 high intensity proton run, from Booster late intensity $60 T P$ to $90 T P$ ( $1 T P=10^{12}$ particles), the BTA transfer efficiency decreases at a rate of 0.45 percentage $/ T P$, from $86 \%$ to $73 \%$. This is estimated using the morning numbers in the Daily Log. The loss increases from 8.4TP to $24 T P$, which is strongly intensity-dependent.

The optics mismatch from the transfer line to the AGS ring is believed for causing emittance blow-up, and hence the beam loss. Several facts, however, suggest that this might not be the dominant factor. Firstly, by varying the quardrupole setting in the BTA line, the optics downstream the line can be changed in a quite wide range. The beam loss pattern seems not much affected. Secondly, in the optics mismatch effect, the most significant factor is the mis-steering. The adjustment of the downstream BTA line vertical steering magnets, however, is not among the most sensitive ones in the tuning. In the third, it is less convincing to correlate the optics mismatch effect with the strongly intensity-dependent loss pattern.

It is known that the beam vertical emittance in the Booster late is strongly intensitydependent. Therefore, the limited BTA vertical aperture induced scraping at F6 [12] can be blamed for the strongly intensity-dependent loss in the BTA transfer. However, the detected loss is not quantitatively proportional to that large amount of loss [13].

The AGS slow bump and fast kicker are also not the strongly intensity-dependent sources of loss.

On the other hand, let us have a close look at the space charge induced incoherent tune shift at the AGS injection.

For the Booster late intensity of $90 T P$, the beam intensity is $84 T P$ by arriving the AGS ring, which means that the AGS injection loss accounts for about $3 / 4$ of the total BTA transfer loss. This number is obtained based on extensive investigations including the calibrations of, and the experiments with, the loss monitors in the Booster, the BTA line, and in the AGS, see $[1,13,14]$. Also included are other informations from the current transformers in the BTA line and in the AGS ring.

The ratio of the bunch length over the RF period is about $B=0.3$, for the first turn in the AGS. This implies that the bunching factor, defined as the ratio of average beam current over the peak current, is $B_{f}=B / 1.5=0.2$. The phase mismatch and the bunch to bucket mismatch produce both dipole and quadrupole oscillations in the injection. The use of VHF dilution can effectively enlarge the bunch length to $B=0.6$, or $B_{f}=0.4$, but this takes a few tens of $m s$, and, therefore, is not relevant to this study.

Finally, it is believed that at high intensity both horizontal and vertical beam emittances are about $\epsilon_{N, 95 \%}=60 \pi \mu m r[12]$.

Using equation (11), therefore, we get the Laslett tune shift at the AGS injection,

$$
\Delta \nu \approx 0.79
$$

The vertical bare tune in the AGS injection porch is 8.85 . This means that the Laslett tune shift has been depressed beyond the half integer by 0.44 , or $56 \%$ of the shift. This can be compared with $22 \%$ of PSR, $24 \%$ of CPS, $32 \%$ of PSB, and $21 \%$ of Fermilab Booster. The emittance growth under this condition is inevitable, and also it is likely that this growth is accompanied by turbulent envelope oscillations.

The estimated beam emittance in the late AGS injection porch is about $\epsilon_{N, 95 \%}=100$ $\pi \mu m r$, for both vertical and horizontal. The horizontal one could be larger. Note that 
on paper the AGS vertical acceptance is larger, at about $150 \pi \mu m r$. However, the real acceptance is believed to be in the vicinity of $100 \pi \mu m r$. In the pre-Booster AGS high intensity proton run of 1985 [15], the vertical beam emittance was $\epsilon_{N, 95 \%}=30 \pi \mu m r$, which implies the same beam size as we have now. So, the real AGS vertical aperture has not changed since. In 1985, however, the beam horizontal emittance was twice as much as the vertical one. Therefore, in present run the AGS horizontal acceptance is probably not filled, at least after the injection bump is collapsed.

If the space charge effect is a dominant factor in the AGS injection beam loss, what happens? Firstly, the loss pattern will be strongly intensity-dependent, and it will be less sensitive to the optics mismatch. Secondly, the AGS bare tune at the injection will be very sensitive. In practice, this is true for the vertical. For the horizontal, it is less sensitive, probably because of some room in the horizontal aperture. Thirdly, the Booster bunch length at extraction will be very sensitive to this loss. On this aspect, the associated momentum spread induced dispersion type loss in the transfer line is also involved. For the last one, the Booster beam emittance will be very sensitive to the AGS injection loss. This has not been observed, because the beam emittance has been limited in the BTA transfer line at high intensity by the beam scraping.

All of these are more or less agreeable with the observations.

Among several low energy proton synchrotrons operated with the ratio of emittance over acceptance of 1, i.e., the AGS Booster, the PS Booster, and also the pre-Booster AGS, the present AGS is the only one that is receiving bunched beams. Therefore, the situation at the AGS injection is unique. The loss associated with the space charge effect can come from two mechanisms. One is that when the beam emittance approaches the machine acceptance, the scraping on the vacuum chamber causes beam loss. The second one could be more important for the AGS. Once the bunched beam enters the AGS ring, with the maximum tune shift depressed beyond the half integer by $56 \%$, a large amount of particles perform betatron oscillations with large amplitudes, due to the beta function distortion. Two things follow: massive beam loss and the rapid emittance blow-up.

\section{References}

[1] L.A. Ahrens, AGS Studies Report, No.349, Aug. 1996.

[2] F. Sacherer, Ph.D Thesis, UCRL-18454, Lawrence Radiation Laboratory, 1968.

[3] S. Machida, Nucl. Inst. Meth, A-309, p.43, 1991.

[4] S. Machida, Proc. Particle Accelerator Conf. p.3224, 1993.

[5] D. Neuffer et al. Proc. Particle Accelerator Conf. p.1893, 1991.

[6] R. Cappi et al. Proc. Particle Accelerator Conf. p.171, 1991.

[7] R. Cappi et al. Proc. Particle Accelerator Conf. p.3570, 1993.

[8] J.P. Delahaye et al. Proc. 11th Int. Conf. High Energy Acc. p.299, 1980.

[9] C. Ankenbrandt and S.D. Holms, Proc. 1987 IEEE Particle Accelerator Conf. p.1066, 1987. 
[10] L.J. Laslett, Proc. of 1963 summer Study of Storage Rings, BNL-Report 7534, p. 324, 1963.

[11] S.Y. Zhang, T. Roser, and W.T. Weng, AGS Tech. Notes, No.449, Oct. 1996.

[12] S.Y. Zhang, AGS Studies Report, No.350, Aug. 1996.

[13] L.A. Ahrens, AGS Studies Report, No.347, Aug. 1996.

[14] L.A. Ahrens, AGS Studies Report, No.348, Aug. 1996.

[15] E. Raka et al. IEEE Trans. Nucl. Sci. NS-32, No.5, p.3110, 1985. 
